\title{
For all the benefits of modern medicine, is the population at large becoming healthier?
}

\section{Mark O Mathews* \\ Sunflower Trust, 10 Guildford Park Road, Guildford, Surrey GU2 7ND, UK}

For thousands of years people throughout the world have looked for ways to discover and address the causes of human pain and dysfunction. In nearly all cultures people have found physical, nutritional, mental, emotional and spiritual ways of helping themselves out of difficulties, many of which are as valid as ever but not being able to patent and make money, many of them have been lost or ignored in our commercially driven culture.

The health benefits of walking and immersing yourself in nature and fulfilling a goal has been completely vindicated through science. Science is a method of enquiry, which is not about being completely materialistic. The process of connecting and re-connecting helps us to be more fully in touch with deeper aspects of ourselves ${ }^{1}$.

Relaxation has been shown to reduce the flight fight response that is mediated by the Sympathetic Nervous System which is conducive to anxiety, stress and inflammation. Such response is the underlying process that is involved in every kind of illness.

There is significant experimental evidence showing that if you remember bad things, and ideas that make you feel angry, your health and behaviour will tend to deteriorate. In contrast to this, people who focus on things for which they feel grateful are much happier, more sociable and fun to be around. Positive attitudes can even help people be more productive in their work and study.

The only certainty is change. One could even be grateful for being fired from a job. It could offer a chance for a new beginning for an even brighter future. Life is an adventure. If everything were predictable it would be very boring.

In my opinion, the digital world may well have increased communication in a limited way for some purposes but in many other ways it has had the effect of alienating people even further away from normal, personal, social relationships pertaining to healthy social communities.

We are all driven by the need to feel good and to avoid the opposite. There are only a few ways in which we can all live together successfully. The qualities natural selection appears to have favoured, because they work, and are based on what we could call civilised values. Behaviours which relate to aspects of communication, morality, compassion, truth and fairness, empathy and mutual respect seem to have worked best; they are found to be extraordinarily similar in nearly all the successful civilisations throughout history.

The ability to heal comes from the innate tendency of all living entities to be whole given the right environment. All living species will

${ }^{1}$ Sheldrake, Rupert (2017) Science and Spiritual Practice Science and Spiritual Practices:
Transformative experiences and their effects on our bodies, brains and health. Coronet Publication.
Punsformative tend to reach out to find this optimum condition for survival, depending on the special ecological niche in which through natural section they have become specialised. In human beings, the dimensions which contribute to such an environment include physical, physiological, mental, emotional, social, cultural and spiritual factors.

Long before the existence of laboratories, as well as using their five senses, people relied more on their innate instincts and intuition to find ways of living in the environments in which they found themselves. Ultimately this is all provided as part of the greater universe by the world ecosystems: the sun, the earth, the air, the water and all the related plants and animals that sustain the complex interactive living ecological systems of the earth.

According to the National Geographic Magazine (November, 2005), there are areas in the world where people following certain life styles live longer healthier lives than in other places. They are called "Blue Zones". They include people of different races and regions. They all tend to have certain things in Common:

1. They have strong family and community values

2. They are mostly physically active every day

3. They sleep more and live their lives in happy relaxed way with a positive attitude

4. They eat mostly natural locally produced fruit vegetables and herbs and meat in moderate amounts

5. They have spiritual values that relate to nature and their surroundings as a whole

6. They do not smoke or consume alcohol

We have been privileged to have inherited a heaven here on earth ranging from the smallest micro-organisms to the largest wales. If we are to have a future to look forward to, it all needs to be loved, appreciated, respected and cared for with gratitude and the greatest of reverence.

It seems that this is now all being disrupted by overpopulation and a contemporised mechanistically restricted way of thinking. In an increasingly complex technical world, the demand for people with the specific skills needed to understand and work within different segments of this economy has resulted in there being more specialists of different

*Correspondence to: Mark O Mathews, Sunflower Trust, 10 Guildford Park Road, Guildford, Surrey GU2 7ND, UK, E-mail: markomathews@aol.com

Received: June 02, 2018; Accepted: June 19, 2018; Published: June 21, 2018 
kinds. The result of this has been that many of them seem to have lost touch with the more holistic view of how everything is interconnected. All the different elements fit together in the broader context of the whole ecosystem that, to be stable, has to remain balanced over time. It is a fact that all life involves the continuous interaction and transformation of energy and information on many different levels over time. We do not live in a vacuum. Nothing happens in isolation. It seems as though society on an economic, political and social level is driven by:

1. The short term, short sighted, selfish pursuit of material wealth and power.

2. International corporate bodies which have no loyalty to the general public or any particular country

3. A growing number of people who have now been educated and conditioned to think of the short term benefits that materialism has brought to relatively limited number of people in the world at a great cost to the greater environment and many other people throughout the world.

Logically this could be regarded as retrograde step. In historical terms, it has put the whole future of the world and mankind, together with its ecological life sustaining systems, at risk.

Most people will visit their Doctor because they are suffering from unpleasant symptoms of one kind or another. We should all be grateful for the amazing technical advances in general medicine and micro surgical procedures producing less collateral damage. Happily most people visiting their GPs with a symptom do not have a life threatening illness. Very often the descriptive name will be given to a symptom is then referred to as if it were a diagnosis. Giving different people the same descriptive label, as if they were all the same, can be misleading. Most people will suffer from the symptoms that they display for their own unique combination of reasons. Nonetheless, they will often be prescribed a drug to repress the symptom.

The average elderly patient is taking more than five prescription medications; the average nursing home patient is taking seven medications. There is increased risk for adverse drug-drug interactions that accompanies high levels of polypharmacy among this patient population.

Medicine is now the third largest cause of death: killing more than 500,000 Europeans and Americans every year ${ }^{2}$. Given this scale of premature death, one seldom hears of many medical authorities being held to account. I wonder why?

We are privileged in this country to have a health service. But much of the ill-health that is putting pressure onto the NHS health is environmentally determined by different aspects of life style. One's health should first of all be regarded as a personal responsibility. We certainly do need to have a safety blanket for those who fall through the net. But why should hard working sensible people who take personal responsibility for looking after themselves properly by eating appropriately, taking exercise and being sensible about managing their affairs have to pay more tax to make up for the irresponsible behaviours of others?

A great deal is said about the pressures on the NHS because people are living longer and the proportion of aging population is increasing. Even the bible records the age "threescore year and ten". This was regarded as normal two thousand years ago. In my student years, under the supervision of the Kenya Range Commission, I lived for three months in the bush with the Maasai near Emali in Kenya. I was

${ }^{2}$ SAFER MEDICINES Registered Charity 1039411 PO Box 122. Kingsbridge.TQ7 9AX able to do this with the help of an academic scholarship from Edinburg University, as an ecologist. I was studying the effect of them and their environment, living as they had done for thousands of years. In that small area there were seven people over 100 years old, something that it was possible to verify. I met one of them on his way to a wedding. $\mathrm{He}$ still had 100 miles to walk.

Up until recent times, the biggest factor that reduced the average age of people living in this country was environmental; particularly for the less well-off working people. Poor diet, inadequate housing, lack of clean water, inadequate drainage, retrograde sewage systems and unhealthy working conditions. Civil engineering, availability of a wider choice of affordable foods, improved housing, better hygiene and legislation on health and safety condition for work have accomplished a great deal more to prolonging life in this country, compared to the NHS, with the exception of antibiotics.

There is of course a place for everything, but as civilised rational human beings, it should be possible to find a sensible balance. Human beings have not changed in thousands of years, but in more recent times, driven by commercial enterprises, our lives and our environment have. Our food is no longer natural; farmers are paid by the look and weight of the food they produce, not the nutritional content. Humans are designed for movement and yet we tend not to perform as much physical exercise. The difference between sitting for 3 hours a day and 6 hours a day can take three years off your life ${ }^{3}$.

Since most people have had the convenience of electric lights, TV's and computers in their homes, they tended to stay up more and get less sleep. For all the convenience that the modern world has to offer, there are also some down sides. These include such things as:

\section{- Information overload}

- Exposure to growing amounts of Electromagnetic smog

- Exposure to millions of synthetic toxic chemicals in the food, in the air, what we touch or put onto our skin, and the millions of thing with which we are surrounded

- The perception by some of the need to keep up with the Joneses

- The overabundance of choices

- Greater demands being made on people's time and attention

- Social and economic pressures

- Being more spread out and commuting greater distances between home, work, family and friends

Such ongoing demands can progressively result in people suffering from much greater levels of stress. This can have the effect of undermining the immune system, which results in many of them becoming more vulnerable to problems of different kinds.

We are paying a heavy price for the delusionary pursuit of never ending growth in a throw-away society. With advances in science, we already have the means and ability to build a zero carbon economy and to be able to manage the resources of the world in a caring sustainable way in the long term interest of health and humanity.

Symptoms are usually the sign that the body is out of balance and its innate safety margin has been breached. This is usually the result of a combination of factors. Simply repressing symptoms without dealing

\footnotetext{
"Nutritional Management of age related declines in musculoskeletal health". Professo Adam Cunliffe, Whittington Education Centre Whittington Hospital London, 18 November 2017
} 
with the underlying causes will eventually result in the development of more chronic illness for which there is no complete cure. This is one of the reasons why the NHS is becoming overwhelmed. It is the largest industry in this country employing 1.7 million people (Telegraph, 20 March, 2012). Despite being so inefficient, it seems to have taken on the status of a kind of a 'sacred cow' which nobody dares question.

Currently, in the media, there have been a number of reports emphasising the growth in the problems of mental health. According to a five year forward view for mental health ${ }^{4}$, one in four adults experiences at least one diagnosable mental health problem in any given year. People from all walks of life can be affected, and at any point in their lives, including new mothers, children, teenagers, adults and older people. Mental health problems represent the largest single cause of disability in the UK. The cost to the economy is estimated at $£ 105$ billion a year - roughly the cost of the entire NHS.

Sometimes these issues are reported as if the mind was in some way completely separate from the body; when, in fact, we experience our feelings through our body. Prescribing more mind altering drugs will very often create bigger problems in the long run.

\footnotetext{
${ }^{4}$ The independent Mental Health Taskforce to the NHS in England, February 2016
}

Much of the media seems to have been driven from behind the scenes by powerful lobbies of commercial interests who have also invested heavily in advertising and other kinds of persuasive and commercially profitable propaganda. It has reached a point where much of the population has been persuaded to believe in very simplified, mechanistic approaches to health and disease which in many ways is proving to be extremely expensive, inefficient and dangerous.

Health is the best defence against disease. It is the result of the harmonious communication and cooperation between all aspects of a human being. Healthy people have a safety margin that enables them to cope with the day-to-day stresses of life.

Using Applied Kinesiology (www.icak.com) it is possible to help people to be more in touch with their own innate perfection. Using this form of Functional Neurology in measured ways, in combination with a range of natural medicine, it is possible for properly trained practitioners to contribute to people's health, wellbeing and success in school, business and life as a whole in an extremely efficient cost effective way.

Copyright: (C2018 Mathews MO. This is an open-access article distributed under the terms of the Creative Commons Attribution License, which permits unrestricted use, distribution, and reproduction in any medium, provided the original author and source are credited. 